
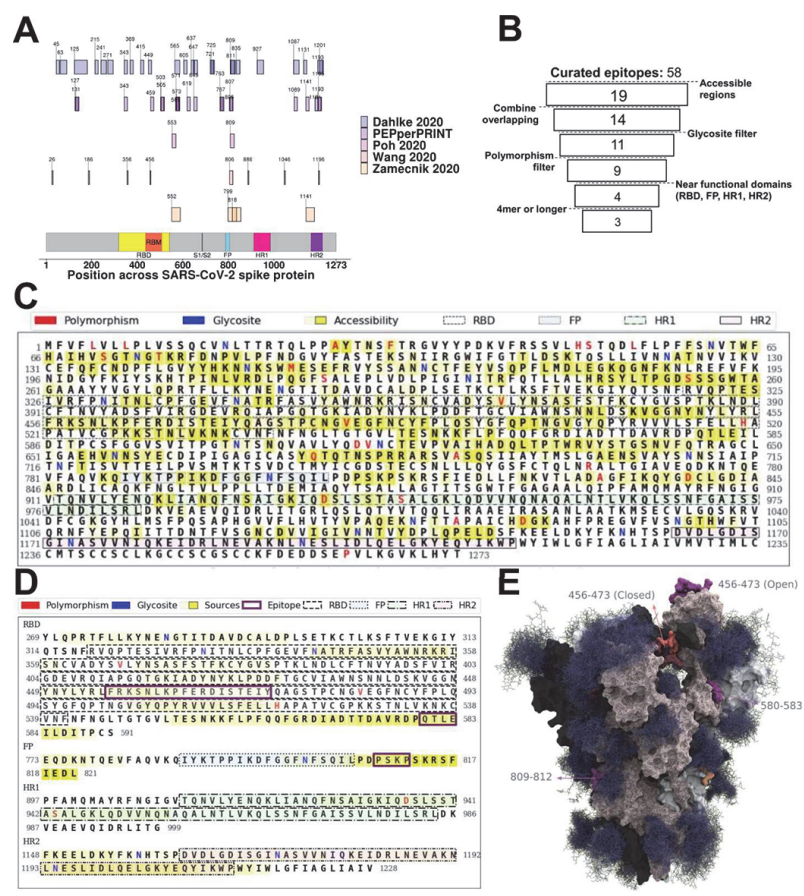

Abstract 478 Figure 5 Selection of SARS-CoV-2 B cell epitope regions. (A) SARS-CoV-2 linear B cell epitopes curated from epitope mapping studies. $\mathrm{X}$-axis represents amino acid position along the SARSCoV-2 spike protein, with labeled start sites. (B) Schematic for filtering criteria of B cell epitope candidates. (C) Spike protein amino acid sequence, with overlay of selection features prior to filtering. Polymorphic residues are red, glycosites are blue, accessible regions highlighted in yellow. The receptor binding domain (RBD), fusion peptide (FP), and HR1/HR2 regions are outlined. (D) Spike protein functional regions (RBD, FP, HR1/2) amino acid sequences, with residues colored by how many times they occur in identified epitopes. Selected accessible sub-sequences of known antibody epitopes highlighted in purple outline. (E) S protein trimer crystal structure with glycosylation, with final linear epitope regions highlighted by color
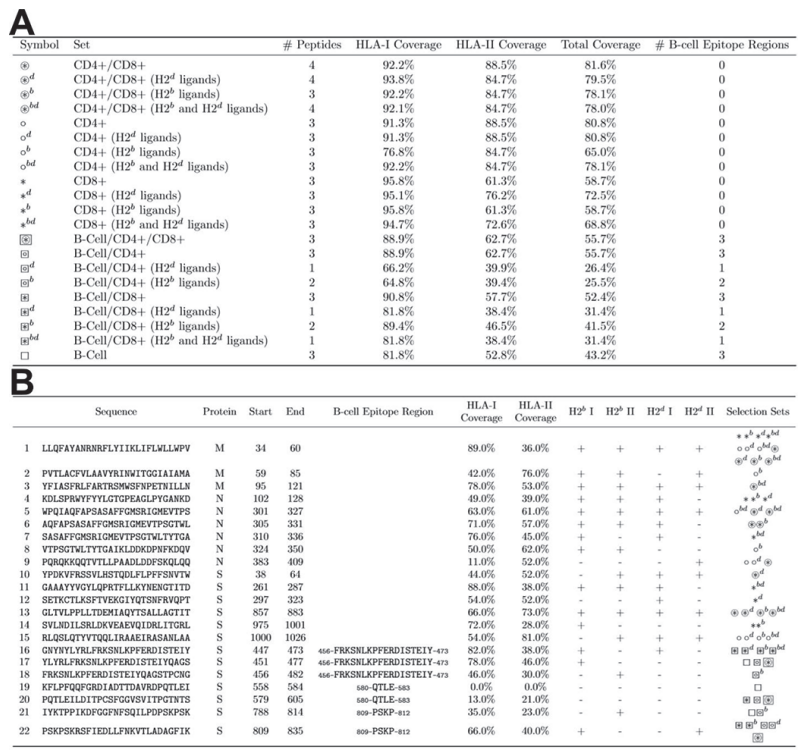

Abstract 478 Figure 6 T cell and B cell vaccine candidates. (A) 27mer vaccine peptide sets selecting for best CD4+, CD8+, CD4+/CD8 + , and B cell epitopes with HLA-I, HLA-II, and total population coverage. (B) Unified list of all selected 27 mer vaccine peptides. Vaccine
A

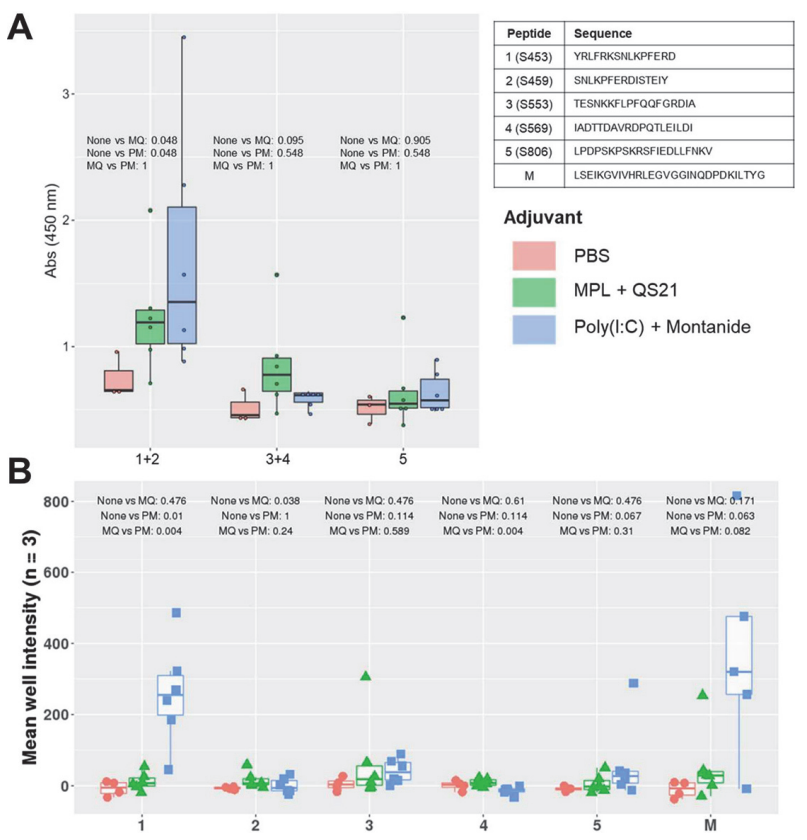

Abstract 478 Figure 7 Immunogenicity of murine-compatible peptide vaccines. (A) ELISA result: peptides derived from three $B$ cell vaccine candidate regions were coated on peptide capture plates, either in combination by overlapping core epitopes $(1+2$ and $3+4)$ or alone (5). (B) ELISpot results: splenocytes from animals vaccinated against predicted B cell epitopes (1-5) or measles peptide control (M; adapted from Obeid et al. 1995). Each point represents the average of technical triplicates, background subtracted against no-peptide control. (A\&B) Colors represent adjuvant used for vaccination. P-values shown above each graph represent pair-wise Mann-Whitney u-test

Conclusions A peptide vaccine targeting B cells, CD4+ T cells, and CD8 $+\mathrm{T}$ cells in parallel may prove an important part of a multifaceted response to the COVID-19 pandemic. Adapting methods for predicting tumor-specific antigens, we presented a set of peptide candidates with high overlap for $T$ and $B$ cell epitopes and broad haplotype population coverage, with validation of immunogenicity in murine vaccine studies.

Acknowledgements The authors appreciate funding support from University of North Carolina University Cancer Research Fund (AR and BGV), the Susan G. Komen Foundation (BGV), the $\mathrm{V}$ Foundation for Cancer Research (BGV), and the National Institutes of Health (CCS, 1F30CA225136). We would like to thank members of the \#DownWithTheCrown Slack channel for helpful discussion and feedback.

http://dx.doi.org/10.1136/jitc-2020-SITC2020.0478

\section{A LYMPH-NODE TARGETED AMPHIPHILE VACCINE INDUCES POTENT CELLULAR AND HUMORAL IMMUNITY TO SARS-COV-2}

Martin Steinbuck*, Peter DeMuth, Lochana Seenappa, Christopher Haqq, Aniela Jakubowski, Lisa McNeil. Elicio Therapeutics, Boston, MA, USA

Background The SARS-CoV-2 pandemic's public health, economic, and social impacts mandate urgent development of effective vaccines to contain or eradicate infection. To that end, we evaluated a novel amphiphile (AMP) vaccine adjuvant, AMP-CpG, composed of diacyl lipid-modified CpG, admixed with the SARS-CoV-2 Spike-2 receptor binding domain (Spike $\mathrm{RBD}$ ) protein for immunization (ELI 005) in two mouse 
models. AMP immunogens are efficiently delivered to lymph nodes, where innate and adaptive immune responses are generated.

Methods Female, 6 to 8-week-old C57BL/6J and BALB/c mice and 37-week-old $\mathrm{C} 57 \mathrm{BL} / 6 \mathrm{~J}$ mice received two or more doses of benchmark (alum or CpG) or AMP-modified vaccines, comprised of Spike RBD protein and AMP-CpG adjuvant, subcutaneously injected into the tail base in two-week intervals. Antigen was dose spared to determine if AMP-CpG would maintain the immune response. Cellular immune responses were determined via ELISpot analysis of IFN $\gamma$ production by splenocytes, intracellular cytokine staining of peripheral blood and lung-resident T-cells, and flowcytometric bead array analysis of Th1/2/17 cytokines. Humoral immune responses were determined via blood serum ELISAs to determine sera antibody binding titers, and pseudoviral neutralization assays for comparison to human convalescent serum.

Results Compared to alum, AMP immunization induced 29fold higher antigen-specific $\mathrm{T}$ cells which produced multiple Th1 cytokines and trafficked into lung parenchyma. Antibody responses favored Th1 isotypes (IgG2bc, IgG3) and potently neutralized Spike-2-ACE2 receptor binding, with titers $>100$ fold higher than the natural immune response from convalescent COVID-19 patients; responses were maintained despite 10 -fold dose-reduction in Spike antigen. Both cellular and humoral immune responses were preserved in aged mice.

Conclusions ELI-005 exhibits the qualities of an optimal SARS-CoV-2 vaccine, which should (1) induce robust and durable $\mathrm{CD} 8+$ and $\mathrm{CD} 4+\mathrm{T}$ cell responses, (2) elicit high magnitude neutralizing antibodies, (3) produce Th1 bias in the elicited antibody and $\mathrm{T}$ cell responses, (4) potentially expand pre-existing cross-reactive $\mathrm{T}$ cells, (5) enable dose-sparing of required immunogens to improve the speed and cost of broad vaccination campaigns, and (6) be efficacious in elderly populations. These advantages merit clinical translation to SARSCoV-2 and other protein subunit vaccines.

http://dx.doi.org/10.1136/jitc-2020-SITC2020.0479

\section{PRELIMINARY EVALUATION OF A NOVEL CORONAVIRUS VACCINE (CORVAX) USING ELECTROPORATION OF PLASMID DNA ENCODING A STABILIZED PREFUSION SARS-COV-2 SPIKE PROTEIN ALONE OR WITH TRANSFECTION OF PLASMID IL-12}

${ }^{1}$ Shawn Jensen*, ${ }^{2}$ Christopher Twitty, ${ }^{3}$ Christopher Paustian, ${ }^{3}$ Madelein Laws, ${ }^{3}$ Glenna McDonnell, ${ }^{1}$ Keith Wegmann, ${ }^{1}$ Tarsem Moudgil, ${ }^{1}$ Michael Afentoulis, ${ }^{2}$ Mia Han, ${ }^{2}$ Kellie Malloy Foerter, ${ }^{2}$ David Canton, ${ }^{2}$ Jack Lee, ${ }^{2}$ Bianca Nguyen, ${ }^{2}$ John Rodriguez, ${ }^{2}$ Kim Jaffe, ${ }^{1}$ Brian Piening, ${ }^{1}$ Carlo Bifulco, ${ }^{2}$ Daniel O'Connor, ${ }^{1}$ Walter Urba, ${ }^{1}$ Rom Leidner, ${ }^{3}$ Traci Hilton, ${ }^{1}$ Hong-Ming Hu, ${ }^{1}$ Bernard Fox. ${ }^{1}$ Earle A. Chiles Research Institute, Prov, Portland, OR, USA; '2OncoSec Medical Incorporated, San Diego, San Diego, CA, USA; ${ }^{3}$ UbiVac, Portland, Oregon, Portland, OR, USA

Background SARS-CoV-2 (CoV2) has precipitated a global pandemic and the effectiveness of standard vaccine strategies to induce potent and persistent immunity to CoV2 is in question, particularly for the elderly. This problem is not dissimilar to what we have struggled with in our quest to induce immunity to cancer antigens, where vaccine-induced anti-cancer immune responses can be weak. Here, we describe a novel vaccine approach which leverages electroporation (EP) of a plasmid encoding a prefusion stabilized $\mathrm{CoV} 2$ spike protein
(CORVax). As IL-12 has been shown to augment the efficacy of immunotherapy in aged mice, ${ }^{1}$ we have initiated studies to evaluate if plasmid IL-12 $\left(\mathrm{TAVO}^{\mathrm{TM}}\right)$ can similarly augment anti-CoV2 immune responses in young mice and have planned studies in aged animals.

Methods A prefusion stabilized CoV2 spike plasmid expression vector was constructed, a master cell bank generated and clinical-grade plasmid manufactured. $\mathrm{C} 57 \mathrm{BL} / 6$ and $\mathrm{BALB} / \mathrm{c}$ were vaccinated via intramuscular (IM) and/or intradermal (ID) injection followed immediately by EP of plasmids encoding the CoV2 spike protein with or without plasmid-encoded murine IL-12 on days 1 and 14 or 21. Mice were followed for $>120$ days to assess safety. Splenocytes and serum were harvested at different time points to interrogate virus-specific cellular responses as well anti-spike $\operatorname{IgG} 1 / \operatorname{IgG} 2$ antibody titers. A surrogate viral neutralization test (sVNT) assessed serum blockade of soluble hACE2R binding to immobilized CoV2 spike.

Results Preliminary data shows that EP of CORVax alone or combined with IL-12 was safe. EP of CORVax was able to elicit anti-Spike IgG antibodies (IC50 = 1/2112), as well as IgG antibodies targeting the receptor binding domain of the Spike protein $(\mathrm{IC50}=1 / 965)$ approximately 40 days after the booster vaccination. In 2 of 2 experiments, CORVax combined with IL-12 significantly $(\mathrm{P}<0.0001)$ increased the sVNT titers at 2 months, but this benefit was lost by 3 months.

Conclusions Early preclinical data shows that EP of CORVax can induce IgG responses to CoV2 Spike and the receptor binding domain (RBD) as well as apparent viral neutralizing activity. The addition of IL-12, at least transiently, increased sVNT titer. We plan to investigate alternate vaccine boosting strategies while extending these studies into aged animals and initiate a clinical trial in the near future.

\section{REFERENCES}

1. Ruby CE, Weinberg AD. OX40-Enhanced tumor rejection and effector T cell differentiation decreases with age. J Immunol 2009;182:1481-9. https://doi.org/ 10.4049/jimmunol.182.3.1481.

http://dx.doi.org/10.1136/jitc-2020-SITC2020.0480

\section{IMPACT OF COVID-19 ON CANCER PATIENTS RECEIVING IMMUNE CHECKPOINT INHIBITORS}

${ }^{1}$ Kevin Tyan*, ${ }^{1}$ Ai-Tram Bui, ${ }^{2}$ Anita Giobbie-Hurder, ${ }^{2}$ Isaac Klein, ${ }^{2}$ Michael Manos, ${ }^{3}$ Leyre Zubiri, ${ }^{3}$ Kerry Reynolds, ${ }^{4}$ Shilpa Grover, ${ }^{4}$ Gerald Weinhouse, ${ }^{2}$ Patrick Ott, ${ }^{4}$ Nicole LeBoeuf, ${ }^{2}$ Osama Rahma. 'Harvard Medical School, Somerville, MA, USA; ${ }^{2}$ DanaFarber Cancer Institute, Boston, MA, USA; ${ }^{3}$ Massachusetts General Hospital, Boston, MA, USA; ${ }^{4}$ Brigham and Women's Hospital, Bostom, MA, USA

Background There are conflicting data regarding the vulnerability of cancer patients receiving immune checkpoint inhibitors (ICIs) to COVID-19 infection. ${ }^{1-3}$ In addition, immunerelated adverse events (irAEs) driven in part by cytokine dysregulation could parallel the cytokine storm implicated in COVID-19. We sought to evaluate the impact of COVID-19 infection on irAEs and mortality in cancer patients receiving ICIs.

Methods We performed a retrospective matched cohort study of 25 patients receiving ICIs within one year of a confirmed COVID-19 diagnosis between March 20, 2020 and June 3, 2020 at the Dana-Farber Cancer Institute/Mass General Brigham network. Cases were matched 1:1 with controls without ICI use based on age, sex, and use of non-ICI anti-cancer 